\title{
Methodology for turbulence code validation: Quantification of simulation- experiment agreement and application to the TORPEX experiment
}

\author{
Paolo Ricci, ${ }^{\text {a) }}$ C. Theiler, A. Fasoli, I. Furno, K. Gustafson, D. Iraji, and J. Loizu \\ Centre de Recherches en Physique des Plasmas-École Polytechnique Fédérale de Lausanne, \\ Association EURATOM-Confédération Suisse, CH-1015 Lausanne, Switzerland
}

(Received 10 November 2010; accepted 1 February 2011; published online 16 March 2011)

\begin{abstract}
A methodology for plasma turbulence code validation is discussed, focusing on quantitative assessment of the agreement between experiments and simulations. The present work extends the analysis carried out in a previous paper [P. Ricci et al., Phys. Plasmas 16, 055703 (2009)] where the validation observables were introduced. Here, it is discussed how to quantify the agreement between experiments and simulations with respect to each observable, how to define a metric to evaluate this agreement globally, and-finally-how to assess the quality of a validation procedure. The methodology is then applied to the simulation of the basic plasma physics experiment TORPEX [A. Fasoli et al., Phys. Plasmas 13, 055902 (2006)], considering both two-dimensional and three-dimensional simulation models. [doi:10.1063/1.3559436]
\end{abstract}

\section{INTRODUCTION}

Validation of plasma turbulence codes plays a fundamental role in assessing maturity of the understanding of plasma dynamics and predictive capabilities of simulations. A validation project is a four step procedure. ${ }^{1}$ (i) First, the simulation model needs to be qualified; i.e., it is necessary to establish the applicability of the model hypotheses for the simulated physical phenomenon. (ii) Second, verification of the code is necessary, in order to prove that the code solves correctly the model equations. (iii) Third, simulation and experiment have to be compared considering a number of physical quantities, common to the experimental measurements and simulation results, and analyzed using the same techniques. These physical quantities, denoted as validation observables, should be identified and organized into a hierarchy. This hierarchy is based on the number of model assumptions and combinations of measurements necessary to obtain the observable, i.e., how stringent each observable is for comparison purposes. (iv) Fourth, agreement between simulations and experiments needs to be quantified by using an appropriate composite metric, $\chi$. This $\chi$ should combine the results of the comparison of all the observables, taking into account position in the hierarchy and precision. Its purpose is to quantify the overall agreement between experiment and simulations. The metric $\chi$ should be complemented by an index, $Q$, which assesses the quality of the comparison. Practically, $Q$ provides an indication of the number of observables that have been used for the validation and the strength of the constraints they impose. While model qualification and code verification [points (i) and (ii) of the validation guidelines] are now routinely considered in plasma physics (see Ref. 2 for some examples) and their methodology has been formulated in considerable detail, only recently has the plasma physics community approached a rigorous methodology for establishing the validation observables and the comparison metric.

\footnotetext{
${ }^{a)}$ Electronic mail: paolo.ricci@epfl.ch.
}

In a previous paper, ${ }^{3}$ we addressed the analysis of point (iii). Focusing on observables related to Langmuir probe measurements, we considered a number of physical quantities that can be used as observables for experiment/ simulation comparison. We classified the observables according to a hierarchy that sums the number of model assumptions and measurement combinations used to obtain an observable from experimental measurements and simulation results. The more assumptions needed, the less stringent the comparison with respect to this observable; and, thus, its weight in the evaluation of the agreement between experiment and simulation should be decreased.

In this paper, we focus on point (iv) of the validation procedure, i.e., on the definition of a metric to quantify the agreement between experiment and simulation. The establishment of a metric involves three steps. First, relative to each observable chosen to perform the comparison, one has to quantify the agreement between experiment and simulation. Second, the levels of agreement relative to each observable have to be combined together to form the composite metric $\chi$ : This process requires that each observable is weighted correctly. The goal is to quantify the global agreement between experiment and simulations with a single index ( $\chi$ is defined such that the lower its value, the better the agreement). Third, the quality of the comparison, $Q$, has to be estimated. This index qualifies the assessment of the agreement between experiment and simulation. We remark that, in order to be meaningful, the comparison between experiments and simulations should be carried out across a scan in the plasma parameters. We also remark that the validation procedures should remain simple. The goal is not mathematical rigor, but a useful tool that can be easily applied in order to compare models and assess their goodness and their limitations.

We believe that the proposed methodology can be easily applied to discriminate among models since a smaller $\chi$ corresponds to a model or a code that provides a better global representation of the physical phenomena at play. Moreover, 
the proposed methodology is very useful to check how the agreement varies with the control parameters. In fact, the increase of $\chi$ that follows from a variation of a control parameter points out the presence of a regime where a particular model is not appropriate presumably because at least one physical phenomenon is not well captured. Thus, the most direct use of the proposed validation methodology is related to determining which code has to be used and in which parameter regime. On the other hand, while it is relatively easy to discriminate among models and the different parameter regimes, it is much more difficult to judge a model in absolute terms. We believe that this question cannot be approached without considering the quantity that the code has to predict within a given tolerance. In fact, once the best code is identified as the one with the lowest $\chi$, one has to focus on the observable of interest, and a code can be considered validated for that observable if the distance between the experimental and simulation data falls within the given tolerance. We note that there is the possibility that, among two codes, the code with the highest $\chi$ better matches the experimental data with respect to the observable of interest; if this happens, we believe that the code with the smallest $\chi$ has to be preferred, nevertheless, since a smaller $\chi$ indicates a better global representation of the physical phenomena into play.

Herein, we first discuss the validation methodology, and then we apply the established procedure to the analysis of the basic plasma physics experiment TORPEX. ${ }^{4}$ Owing to its detailed diagnostics, possibility of parameter scans, and relatively simple configuration, TORPEX is an ideal testbed to perform experiment/simulation comparisons and to investigate the corresponding methodological framework. As detailed later, we perform the comparison between experiments and simulations through a parameter scan, allowing the properties of the TORPEX turbulence to pass from $k_{\|}=0$ mode dominated turbulence to $k_{\|} \neq 0$ turbulence. We validate two models that have been recently developed to simulate TORPEX turbulence: a three-dimensional two-fluid model, able to describe the global evolution of TORPEX plasma, ${ }^{5,6}$ and a reduced two-dimensional two-fluid model, ${ }^{7}$ able to describe only the evolution of $k_{\|}=0$ modes. We show that the validation metric is able to point out that the agreement of the two-dimensional model and the experiment is no longer satisfactory when $k_{\|} \neq 0$ modes are present in the experiment.

The paper is structured as follows. After the introduction, we describe in Sec. II how to quantify the agreement for each observable. The construction of a metric that takes into account all the observables is presented in Sec. III, while in Sec. IV we discuss the quality of the comparison. The application of the methodology for comparison between experiment and simulation is discussed in Sec. V, where we show how it is possible to apply the concepts that have been introduced to the analysis of the basic plasma physics experiment TORPEX. The conclusions follow. In the appendixes, we present the TORPEX experimental setup (Appendix A) and the simulation approach (Appendix B) considered in Sec. V.

\section{QUANTIFICATION OF THE AGREEMENT OF EACH OBSERVABLE}

A key step in the construction of the global metric is the quantification of the agreement between experiment and simulation relative to each observable. As discussed in Ref. 3 , one has to use the same techniques to analyze experimental and simulation data, in order to directly compare the experimental and simulation values of each observable. We denote with $x_{j}$ and $y_{j}$ the values of the $j$ th observable coming from the experimental measurement and the simulation results, respectively. Most of the observables depend on space and time, and typically the value of the observables is given on a discrete number of points. We denote with $x_{j, i}$ and $y_{j, i}$ the values of the $j$ th observable at points $i=1,2, \ldots, N_{j}$ (the present notation will be used for zero-, one-, two-, etc., dimensional observables). For the $j$ th observable, we normalize the distance $d_{j}$ between experiment and simulation with respect to the uncertainty related to these quantities:

$$
d_{j}=\sqrt{\frac{1}{N_{j}} \sum_{i=1}^{N_{j}} \frac{\left(x_{j, i}-y_{j, i}\right)^{2}}{\Delta x_{j, i}^{2}+\Delta y_{j, i}^{2}}},
$$

where $\Delta x_{j, i}$ and $\Delta y_{j, i}$ are the uncertainties, i.e., the rms, related to the evaluation of $x_{j, i}$ and $y_{j, i}$. Since it is possible to argue that the simulations and experiment agree if they fall within the error bars, we define the level of agreement between experiment and simulation for observable $j$ as

$$
R_{j}=\frac{\tanh \left[\left(d_{j}-d_{0}\right) / \lambda\right]+1}{2},
$$

with the meaning that $R_{j}=0$ corresponds to perfect agreement (within the error bars), while $R_{j}=1$ denotes complete disagreement between simulation and experiment. For $d_{j}=d_{0}, R_{j}=0.5$; therefore, $d_{0}$ represents the transition from agreement to disagreement. The value of $d_{0}$ should then correspond to a discrepancy between experiment and simulation that is comparable to their uncertainties. In the case $\Delta x_{j, i}=\Delta y_{j, i}, d_{0} \simeq 1.4$ is the distance corresponding to a discrepancy between experiment and simulation equal to the sum of the experimental and simulation uncertainties. The parameter $\lambda$ instead denotes the sharpness of the transition from $R_{j}=0$ to $R_{j}=1$. The test described in Sec. V shows that the conclusions of a validation exercise are not affected for parameters in the ranges $1 \leq d_{0} \leq 2$ and $0.1 \leq \lambda \leq 1$, and we believe that the values $d_{0}=1.5$ and $\lambda=0.5$ are reasonable choices.

Particular attention should be paid in evaluating the experimental and simulation error bars. In the case of the experiments, we can identify three main uncertainty sources. First, the model of a measuring device provides predictions through which one can infer the physical quantities of interest (e.g., from the I-V curve of a Langmuir probe one can infer plasma properties like $n$ and $T_{e}$ ). Experimental measurements typically do not follow perfectly the model predictions; thus, a fit has to be made in order to evaluate the relevant physical parameters, introducing an uncertainty that we denote with $\Delta x_{j, i}^{\mathrm{fit}}$. Second, a source of uncertainty is due to properties of the measuring device that are often difficult 
to evaluate accurately (e.g., geometry and surface condition of a Langmuir probe). Thus, measurements should be performed with different tools (e.g., Langmuir probes which differ in dimension, surface condition, and electronics). The quantity $\Delta x_{j, i}^{\mathrm{prb}}$ denotes the uncertainty related to the probe properties. Third, the plasmas are not perfectly reproducible due to control parameters that are difficult to set or know precisely (e.g., the vacuum pressure). Experiments should be repeated in order to check the reproducibility of the plasma, while measurements are taken with different measurement devices. The quantity $\Delta x_{j, i}^{\text {rep }}$ is the uncertainty due to the plasma reproducibility, averaged over the different measuring devices. Finally, the use of a finite time data set constitutes a source of uncertainty, $\Delta x_{j, i}^{\text {fin }}$, which can be relevant particularly if high moments of the probability distribution functions (PDFs) are considered as observables and which can be estimated through statistical techniques. One can show that the total experimental uncertainty is given by $\Delta x_{j, i}^{2}=\left(\Delta x_{j, i}^{\mathrm{fit}}\right)^{2}+\left(\Delta x_{j, i}^{\mathrm{prb}}\right)^{2}+\left(\Delta x_{j, i}^{\mathrm{rep}}\right)^{2}+\left(\Delta x_{j, i}^{\mathrm{fin}}\right)^{2}$.

Simulations are also affected by uncertainties resulting from three sources: (i) errors due to the numerics (e.g., due to the limited accuracy of the numerical integration scheme used or due to the finite grid resolution), (ii) errors due to unknown or imprecise input parameters, and (iii) statistical uncertainty due to a finite time series. While errors due to the numerics, $\Delta y_{j, i}^{\text {num }}$, can be estimated through convergence tests, the evaluation of the error related to imperfectly known input parameters, $\Delta y_{j, i}^{\text {inp }}$, requires a sensitivity study, i.e., an investigation of how the model results are affected by the input parameter variations. Usually, the number of input parameters of a turbulence simulation code is quite large, and a complete study of the model response is prohibitive. However, the theory can indicate to which input parameters the results are particularly sensitive. The analysis has then to focus on those. We remark that, in the literature, a number of useful techniques have been proposed to predict the response of a model to variation of simulation parameters using the smallest possible number of simulations (see, e.g., Ref. 8). The uncertainty due to a finite set of data, $\Delta y_{j, i}^{\text {fin }}$, is typically more relevant for the simulation than for the experiment since, because of the computational cost, the time series obtained from the simulations is typically limited to a short time span. Also, in this case, this uncertainty should be evaluated by using statistical techniques. As in the case of the experimental error bars, the three sources of error should be added, such that $\Delta y_{j, i}^{2}=\left(\Delta y_{j, i}^{\text {num }}\right)^{2}+\left(\Delta y_{j, i}^{\text {inp }}\right)^{2}+\left(\Delta y_{j, i}^{\text {fin }}\right)^{2}$. We note that the error bars should not take into account the uncertainties related to model assumptions and/or to combinations of measurements, which are often needed to deduce the comparison observables from the simulation results and the raw experimental data. ${ }^{3}$ Evaluating rigorously those uncertainties is usually very challenging. The idea is to take them into account approximately through the observables primacy hierarchy. More specifically, the higher is the hierarchy level of an observable, the lower is the importance of the observable in the comparison metric.

We finally remark that not all observables can be used for a given comparison. In order for an observable to be considered, it should satisfy the following criteria. First, the observable should be physically relevant, i.e., focus should be put on observables containing the most important theoretical predictions. On the other hand, if a code is specifically used to predict a particular set of quantities, these should be necessarily included as validation observables. Second, each observable should be independent and not be a function of the other observables. For example, if the time average of density, $\bar{n}$, and the time average of the temperature, $\bar{T}_{e}$, are observables, then the pressure, $\bar{p}_{e}=\bar{n} \bar{T}_{e}$, cannot be considered a new observable. However, if $\bar{p}_{e}$ is evaluated thanks to an independent measurement, such that it does not need to be written as a function of the other observables, then $\bar{p}_{e}$ can be considered a new observable. Third, the resolution of multidimensional observables should be sufficient to well describe their variation along all dimensions. For example, the spatial resolution of an observable like the density profile should be sufficient to describe the typical density scale length, $L_{n}$.

\section{METRIC}

The overall level of agreement between simulation and experiment can be measured by considering a composite metric, which should take into account the level of agreement of each observable, $R_{j}$, and weight it according to how constraining each observable is for comparison purposes. This means that the hierarchy level of each observable as well as the level of confidence characterizing the measurement or the simulation of each observable has to be taken into account: The higher the level in the primacy hierarchy and the bigger the error affecting the observable measurement, the smaller the weight of the observable should be. We thus define the metric $\chi$ as

$$
\chi=\frac{\sum_{j} R_{j} H_{j} S_{j}}{\sum_{j} H_{j} S_{j}},
$$

where $H_{j}$ and $S_{j}$ are functions defining the weight of each observable according to its hierarchy level and the precision of the measurement, respectively. The metric thus defined is normalized in such a way that perfect agreement is observed for $\chi=0$ (within the considered observables), while simulation and experiment disagree completely for $\chi=1$.

The definition of $H_{j}$ and $S_{j}$ is somewhat arbitrary. This $H_{j}$ should be a decreasing function of hierarchy levels. The definition we adopt is $H_{j}=1 / h_{j}$, where $h_{j}$ is the combined experimental/simulation primacy hierarchy level (see Ref. 3 for a list of the hierarchy levels relative to Langmuir probe measurements). This definition implies that if no assumptions or combinations of measurements are used for obtaining an observable, then $H_{j}=1$. The quantity $S_{j}$ should be a decreasing function of the experimental and simulation uncertainties. We introduce the following definition:

$$
S_{j}=\exp \left(-\frac{\sum_{i} \Delta x_{j, i}+\sum_{i} \Delta y_{j, i}}{\sum_{i}\left|x_{j, i}\right|+\sum_{i}\left|y_{j, i}\right|}\right),
$$

such that $S_{j}=1$ in the case of zero uncertainty. We remark that, while the composite metric $\chi$ is a very useful tool to provide the overall assessment of the agreement, it is also very important to provide a table, which we will denote as a 
"validation table," with a list of the observables used for the comparison, as well as the values of $d_{j}, S_{j}$, and $H_{j}$ related to each observable. This is useful for two reasons. First, it can reveal if agreement between simulations has been evaluated by considering a relevant set of observables. Second, by studying the agreement of each observable one can infer physical effects missing from the simulations.

\section{QUALITY}

The validation metric should be complemented by an index, $Q$, that assesses the "quality" of the comparison. The idea is that a validation is more reliable with a larger number of independent observables, particularly if they occupy a low level in the primacy hierarchy, and the measurement and simulation uncertainties are low. The quality of the comparison, $Q$, can thus be defined as

$$
Q=\sum_{j} H_{j} S_{j} .
$$

Particular attention should be paid to the interpretation of the $Q$ index since the quality of the comparison unavoidably depends on the choice of the observables used. Careful study of the validation table is therefore necessary to obtain a complete picture of the comparison quality.

A compromise between the single parameter $Q$ and the analysis of the full validation table can be made by using a set of parameters to characterize the quality of the validation. In particular, we note that the quality of a comparison is higher if, e.g., a two-dimensional observable is used for the comparison instead of a one-dimensional observable. Therefore, a set of $Q$ indices, $Q_{n}$, can be introduced in order to take into account separately observables with different dimensionalities, i.e., $Q_{n}=\sum_{j_{n}} H_{j} S_{j}$, where the sum is restricted to the $n$-dimensional observables.

\section{EXAMPLE OF SIMULATION AND EXPERIMENT COMPARISON}

We present an example of comparison between experimental data and simulation results to apply the methodology discussed in the previous sections. The comparison is carried out on the basic plasma physics experiment TORPEX. ${ }^{4}$ This experiment is particularly suited for experiment/simulation comparison, thanks to its detailed diagnostics, the possibility of performing easily parameter scans, and relatively simple geometry, which allows global simulations particularly suited for a detailed comparison.

TORPEX is a simple magnetized plasma, a toroidal configuration of major radius $R$ and height $L_{v}$ where a vertical magnetic field, $B_{z}$, superimposed on a toroidal magnetic field, $B_{\phi}$, creates helicoidal field lines winding around the device. Pressure gradients and magnetic curvature drive a number of instabilities. As discussed in Ref. 6, simulations reveal that there are two turbulent regimes in TORPEX, each primarily driven by a distinct plasma instability: the ideal and the resistive interchange modes. The most obvious difference between the two regimes is the wavenumber along the magnetic field: $k_{\|}=0$ in the ideal interchange case, while $k_{\|} \neq 0$ in the resistive case. The main parameter that controls the transition from one instability to the other is the pitch of the field line, expressed in terms of $\Delta=2 \pi R B_{z} / B_{\phi}$, the vertical return distance of a field line in the poloidal plane, or in terms of $N=L_{v} / \Delta$, the number of field line turns in the device. At low values of $N$, TORPEX dynamics is dominated by the ideal interchange regime. The transition to the resistive regime is expected to occur at $N \simeq 10$, as confirmed experimentally. ${ }^{9}$

The TORPEX experimental setup and the Langmuir probe diagnostics used (HEXTIP, SLP, and TWIN) are described in Appendix A. We consider two simulation models: a two-dimensional two-fluid model that is able to represent only $k_{\|}=0$ modes and a global three-dimensional two-fluid model that describes the evolution of the plasma dynamics in the full TORPEX volume. The details of the simulation models are described in Appendix B.

For the validation example we consider a set of TORPEX plasma configurations with different values of vertical magnetic field, characterized by different properties of plasma turbulence. We analyze four scenarios, characterized by $N=2,4,8$, and 16 , and we carry out simulations using both two-dimensional and three-dimensional models. As validation observables, we use the equilibrium radial profiles at the vertical midplane of density, $\bar{n}$; electron temperature, $\bar{T}_{e}$; electric potential, $\bar{\phi}$; and ion saturation current, $\bar{I}_{\text {sat }}$. We also use: the normalized $I_{\text {sat }}$ fluctuations, $\delta I_{\text {sat }} / \bar{I}_{\text {sat }}$; the $I_{\text {sat }}$ skewness and kurtosis; the value of the vertical and toroidal wavenumbers, $k_{z}$ and $k_{\phi}$; the PDF and the power spectrum density (PSD) of $I_{\text {sat }}$ at the vertical midplane, at the radial point where $\bar{I}_{\text {sat }}$ is equal to $3 / 4$ of its peak value (i.e., in correspondence to a point where we expect to identify more clearly the turbulence properties). We perform the comparison using the toroidal mode number only for the threedimensional simulations since it is not calculated selfconsistently by the two-dimensional model assuming $k_{\|}=0$. The results of the validation are summarized in Table I and are discussed below in detail.

\section{A. Agreement of each observable}

For each observable, the experimental error bar $\Delta x$ is evaluated by repeating the experiments a number of times and comparing the measurements of different probes. The simulation uncertainty, $\Delta y$, is evaluated by performing for each TORPEX scenario a number of simulations where we vary the parameters that we expect to significantly affect the simulations and that are not well known. For the threedimensional simulations, these are the plasma resistivity $\nu$ and the boundary conditions. For the two-dimensional simulation, instead, the sensitivity to the parallel losses is studied. We assume that the error due to the numerics is negligible with respect to the error due to the unknown input parameters.

In Figs. 1-10 we present the observables used for our code validation. The value of the distance $d$ between experiments and simulations with respect to each observable is evaluated and listed in Table I.

In Fig. 1, we plot the radial profile of equilibrium density, $\bar{n}$, at the vertical midplane, as obtained from the experi- 
TABLE I. Validation table for the two- and three-dimensional TORPEX simulations. The values $R$ are evaluated assuming $\lambda=0.5$ and $d_{0}=1.5$.

\begin{tabular}{|c|c|c|c|c|c|c|c|c|c|}
\hline \multirow[b]{2}{*}{ Observable } & \multirow[b]{2}{*}{ Dimensionality } & \multirow[b]{2}{*}{$H$} & \multirow[b]{2}{*}{$N$} & $d$ & $R$ & $S$ & $d$ & $R$ & $S$ \\
\hline & & & & \multicolumn{3}{|c|}{ 2D simulation } & \multicolumn{3}{|c|}{ 3D simulation } \\
\hline \multirow[t]{4}{*}{$\bar{n}$} & 1 & 0.5 & 2 & 1.34 & 0.35 & 0.89 & 0.83 & 0.06 & 0.91 \\
\hline & & & 4 & 1.33 & 0.33 & 0.88 & 3.21 & 1.00 & 0.84 \\
\hline & & & 8 & 3.60 & 1.00 & 0.90 & 1.66 & 0.65 & 0.84 \\
\hline & & & 16 & 7.81 & 1.00 & 0.91 & 2.00 & 0.88 & 0.89 \\
\hline \multirow[t]{4}{*}{$\bar{\phi}$} & 1 & 0.5 & 2 & 1.19 & 0.22 & 0.89 & 3.12 & 1.00 & 0.89 \\
\hline & & & 4 & 3.39 & 1.00 & 0.90 & 1.63 & 0.63 & 0.82 \\
\hline & & & 8 & 6.91 & 1.00 & 0.91 & 0.82 & 0.06 & 0.73 \\
\hline & & & 16 & 7.96 & 1.00 & 0.92 & 1.51 & 0.51 & 0.75 \\
\hline \multirow[t]{4}{*}{$\overline{T_{e}}$} & 1 & 0.5 & 2 & 2.30 & 0.96 & 0.91 & 3.59 & 1.00 & 0.92 \\
\hline & & & 4 & 2.28 & 0.95 & 0.91 & 5.11 & 1.00 & 0.89 \\
\hline & & & 8 & 6.05 & 1.00 & 0.92 & 6.42 & 1.00 & 0.93 \\
\hline & & & 16 & 6.89 & 1.00 & 0.93 & 4.12 & 1.00 & 0.92 \\
\hline \multirow[t]{4}{*}{$\bar{I}_{\text {sat }}$} & 1 & 0.5 & 2 & 0.42 & 0.01 & 0.74 & 0.45 & 0.01 & 0.75 \\
\hline & & & 4 & 0.66 & 0.03 & 0.69 & 2.56 & 1.00 & 0.73 \\
\hline & & & 8 & 3.01 & 1.00 & 0.81 & 1.97 & 0.87 & 0.73 \\
\hline & & & 16 & 4.55 & 1.00 & 0.84 & 1.61 & 0.61 & 0.77 \\
\hline \multirow[t]{4}{*}{$\delta I_{\text {sat }} / \bar{I}_{\text {sat }}$} & 1 & 0.5 & 2 & 4.51 & 1.00 & 0.90 & 3.96 & 1.00 & 0.91 \\
\hline & & & 4 & 7.77 & 1.00 & 0.90 & 7.47 & 1.00 & 0.90 \\
\hline & & & 8 & 5.28 & 1.00 & 0.90 & 7.04 & 1.00 & 0.86 \\
\hline & & & 16 & 5.56 & 1.00 & 0.88 & 4.00 & 1.00 & 0.82 \\
\hline \multirow[t]{4}{*}{$I_{\text {sat }}$ skewness } & 1 & 0.5 & 2 & 3.10 & 1.00 & 0.85 & 3.72 & 1.00 & 0.81 \\
\hline & & & 4 & 3.41 & 1.00 & 0.74 & 2.69 & 1.00 & 0.64 \\
\hline & & & 8 & 4.02 & 1.00 & 0.83 & 2.27 & 0.96 & 0.62 \\
\hline & & & 16 & 3.03 & 1.00 & 0.66 & 1.48 & 0.48 & 0.62 \\
\hline \multirow[t]{4}{*}{$I_{\text {sat }}$ kurtosis } & 1 & 0.5 & 2 & 3.32 & 1.00 & 0.92 & 2.90 & 1.00 & 0.91 \\
\hline & & & 4 & 2.94 & 1.00 & 0.85 & 2.93 & 1.00 & 0.83 \\
\hline & & & 8 & 5.39 & 1.00 & 0.91 & 1.14 & 0.19 & 0.70 \\
\hline & & & 16 & 2.77 & 0.99 & 0.83 & 1.32 & 0.32 & 0.86 \\
\hline \multirow[t]{4}{*}{$I_{\text {sat }} \mathrm{PDF}$} & 1 & 0.5 & 2 & 1.08 & 0.15 & 0.72 & 0.88 & 0.08 & 0.68 \\
\hline & & & 4 & 0.94 & 0.10 & 0.74 & 0.53 & 0.02 & 0.53 \\
\hline & & & 8 & 0.46 & 0.02 & 0.67 & 0.25 & 0.01 & 0.40 \\
\hline & & & 16 & 0.54 & 0.02 & 0.60 & 0.19 & 0.01 & 0.23 \\
\hline \multirow[t]{4}{*}{$I_{\mathrm{sat}} \mathrm{PSD}$} & 1 & 0.5 & 2 & 1.11 & 0.17 & 0.46 & 0.58 & 0.02 & 0.43 \\
\hline & & & 4 & 1.16 & 0.20 & 0.44 & 0.41 & 0.01 & 0.31 \\
\hline & & & 8 & 1.22 & 0.25 & 0.40 & 0.55 & 0.02 & 0.32 \\
\hline & & & 16 & 1.28 & 0.29 & 0.40 & 2.13 & 0.93 & 0.49 \\
\hline \multirow[t]{4}{*}{$k_{z}$} & 1 & 0.5 & 2 & 1.79 & 0.76 & 0.42 & 1.32 & 0.33 & 0.69 \\
\hline & & & 4 & 3.63 & 1.00 & 0.33 & 0.67 & 0.03 & 0.61 \\
\hline & & & 8 & 7.24 & 1.00 & 0.36 & 0.71 & 0.04 & 0.53 \\
\hline & & & 16 & 6.28 & 1.00 & 0.29 & 0.74 & 0.05 & 0.60 \\
\hline \multirow[t]{4}{*}{$k_{\phi}$} & 0 & 0.5 & 2 & $\cdots$ & $\cdots$ & $\cdots$ & 0.25 & 0.01 & 0.92 \\
\hline & & & 4 & $\cdots$ & $\cdots$ & $\cdots$ & 0.50 & 0.02 & 0.86 \\
\hline & & & 8 & $\cdots$ & $\ldots$ & $\ldots$ & 0.64 & 0.03 & 0.86 \\
\hline & & & 16 & $\cdots$ & $\cdots$ & $\cdots$ & 0.13 & 0.00 & 0.91 \\
\hline
\end{tabular}

ment, the three-dimensional simulations, and the twodimensional simulations, for the different values of $N$. The values of $\bar{n}$ are evaluated by sweeping the bias of the Langmuir probes and fitting the obtained values with an I-V curve. It is possible to see good agreement between experiments and simulations for $N=2$; the agreement deteriorates at higher $N$, particularly for the two-dimensional code.
The analysis of the I-V curve also leads to the evaluation of the equilibrium potential, $\bar{\phi}$, and of the electron equilibrium temperature, $\bar{T}_{e}$, which are plotted in Figs. 2 and 3, respectively. The profile of $\bar{\phi}$ is reasonably described by the three-dimensional simulations at high $N$, while the agreement is worse for the $\bar{T}_{e}$ profile. We remark in particular that 

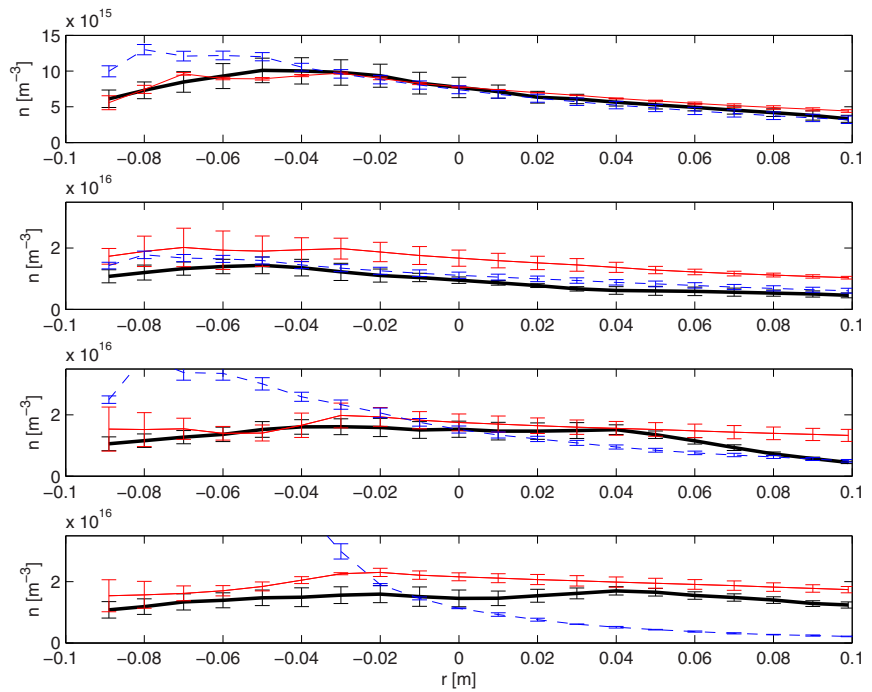

FIG. 1. (Color online) Radial profile of equilibrium density $\bar{n}$, as obtained from the experiment (thick black line), the two-dimensional simulation (dashed blue line), and the three-dimensional simulation (thin red line). The coordinate $r$ denotes the radial distance from the center of the poloidal cross section (i.e., $r=0$ corresponds to a distance of $1 \mathrm{~m}$ from the torus axis); the profiles are evaluated at the vertical midplane. From the upper to the lower panels, the $N=2,4,8$, and 16 scenarios are plotted. The experimental and simulation uncertainties are also displayed.

the simulations are not able to provide a good estimate of the $\bar{T}_{e}$ scale length, probably because of a poor description of the heat losses to the vessel.

By using the Langmuir probes in the $I_{\text {sat }}$ regime, it is possible to obtain the measurement of the $I_{\text {sat }}$ current as a function of time. Its average value, $\bar{I}_{\text {sat }}$, is plotted in Fig. 4. As in the case of the $\bar{n}$ observable, the agreement is good at low $N$ and degrades at high $N$. We have also analyzed the higher moments of the $I_{\text {sat }}$ time trace. A significant discrepancy between simulations and experiments is revealed by the comparison of the turbulence amplitude levels, as displayed by the normalized root mean square value of the $I_{\text {sat }}$ profile in
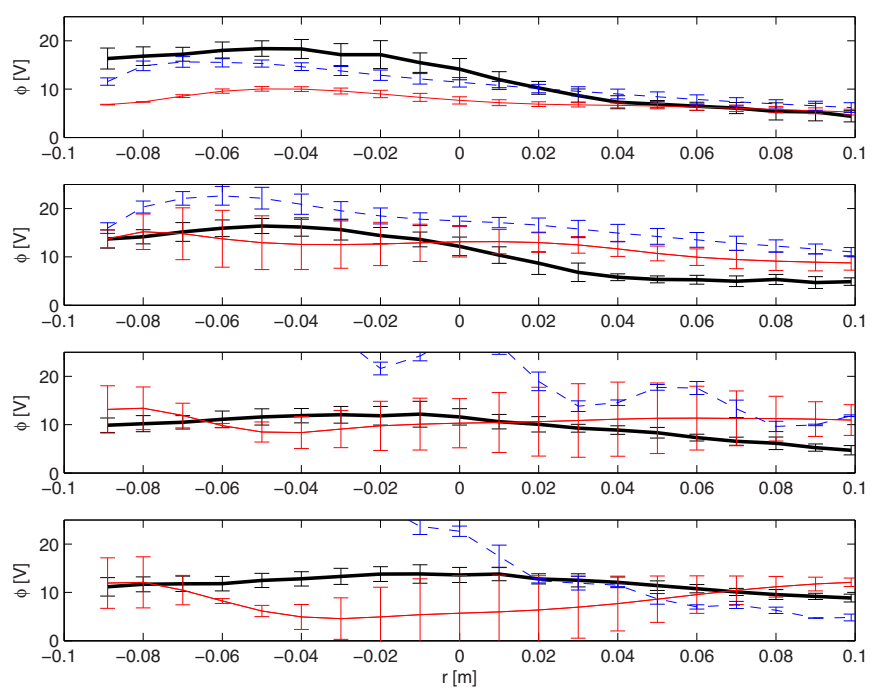

FIG. 2. (Color online) Same as in Fig. 1 for $\bar{\phi}$, the radial time-averaged potential profile at the vertical midplane.
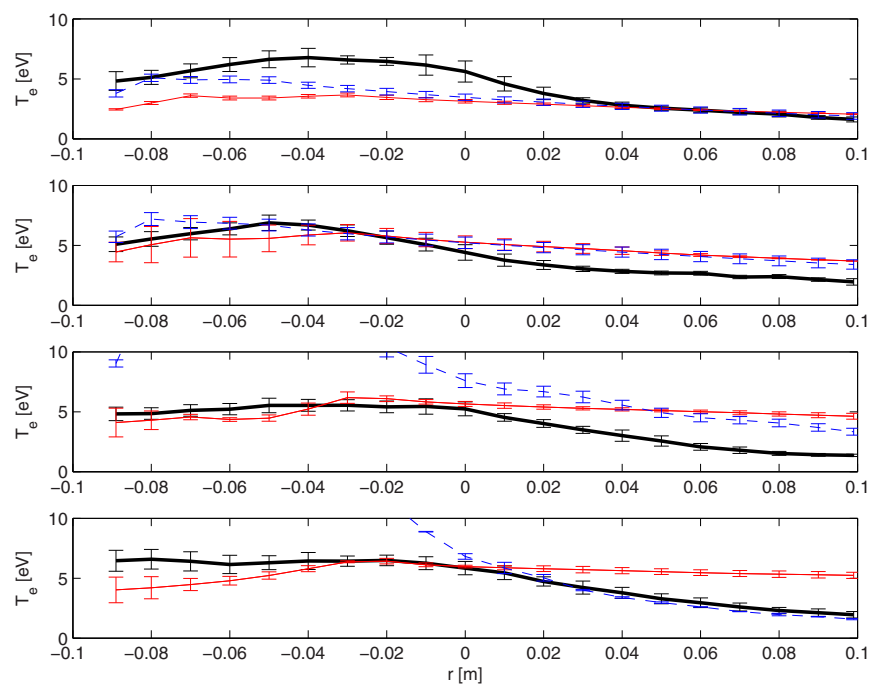

FIG. 3. (Color online) Same as in Fig. 1 for $\bar{T}_{e}$, the radial time-averaged electron temperature profile at the vertical midplane.

Fig. 5, which shows, in fact, that the simulated turbulence amplitude is about a factor of 2 smaller than the experimental one. This suggests the presence of fluctuation sources absent in the simulations or the presence of modes that saturate at a larger amplitude. The normalized third and fourth moments of the $I_{\text {sat }}$ time trace, the skewness and the kurtosis, are plotted in Figs. 6 and 7. These quantities are particularly important for the study of the universal properties of turbulence. ${ }^{10}$ Finally, in Figs. 8 and 9, we plot the PDF and the PSD of the $I_{\text {sat }}$ time trace at the radial point where $\bar{I}_{\text {sat }}$ is equal to $3 / 4$ of its peak value. The PDF and PSD are normalized to the same area for experimental and simulation data, and the PDFs are rescaled so that the $I_{\text {sat }}$ time traces have the same average values and variance. We note that the PSD analysis reveals that the difference between the experimental and simulated turbulence levels is not due to coherent fluctuations at a particular frequency.
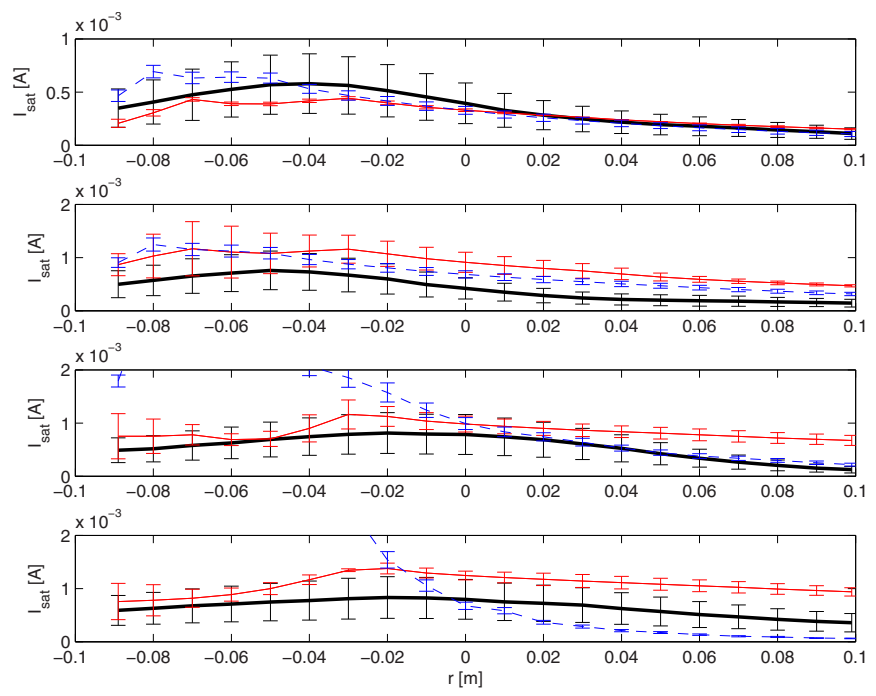

FIG. 4. (Color online) Same as in Fig. 1 for $\bar{I}_{\text {sat }}$, the radial profile of the time-averaged ion saturation current at the vertical midplane. 

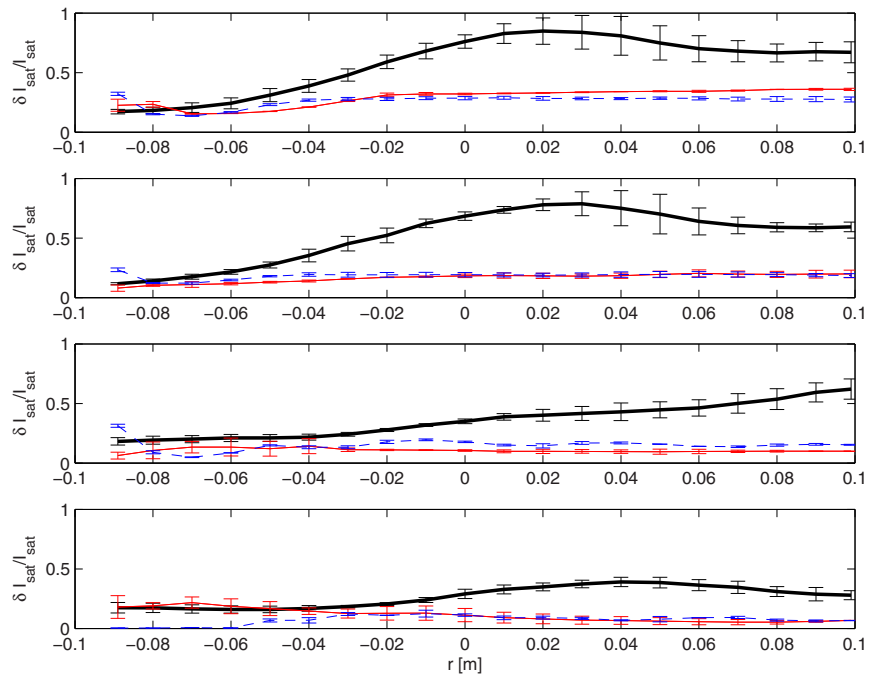

FIG. 5. (Color online) Same as in Fig. 1 for $\delta I_{\text {sat }} / \bar{I}_{\text {sat }}$, the radial profile of the normalized standard deviation of ion saturation current.

The last two observables considered in the present analysis are related to the turbulence wavenumbers, in particular, the vertical and toroidal wavenumbers, $k_{z}$ and $k_{\phi}$. These are measured by using the Fourier transform HEXTIP data for $k_{z}$, and the cross-correlation technique described in Ref. 11 for $k_{\phi}$. The $k_{z}$ observable is plotted in Fig. 10, showing a reasonable agreement for the three-dimensional simulations and complete disagreement for the two-dimensional ones at high $N$. On the other hand, the $k_{\phi}$ comparison can only be carried out for the three-dimensional simulations. We have focused on the evaluation of the toroidal wavenumber corresponding to the dominant mode. The experimentally measured toroidal wavenumbers are $k_{\phi}=1.02,1.08,0.047$, and $0.006 \mathrm{~m}^{-1}$, respectively, for $N=2,4,8$, and 16 . The simulations show $k_{\phi}=1,1,0$, and $0 \mathrm{~m}^{-1}$, thus revealing that the turbulence wavenumbers are well described by the threedimensional simulations. We note that the transition from
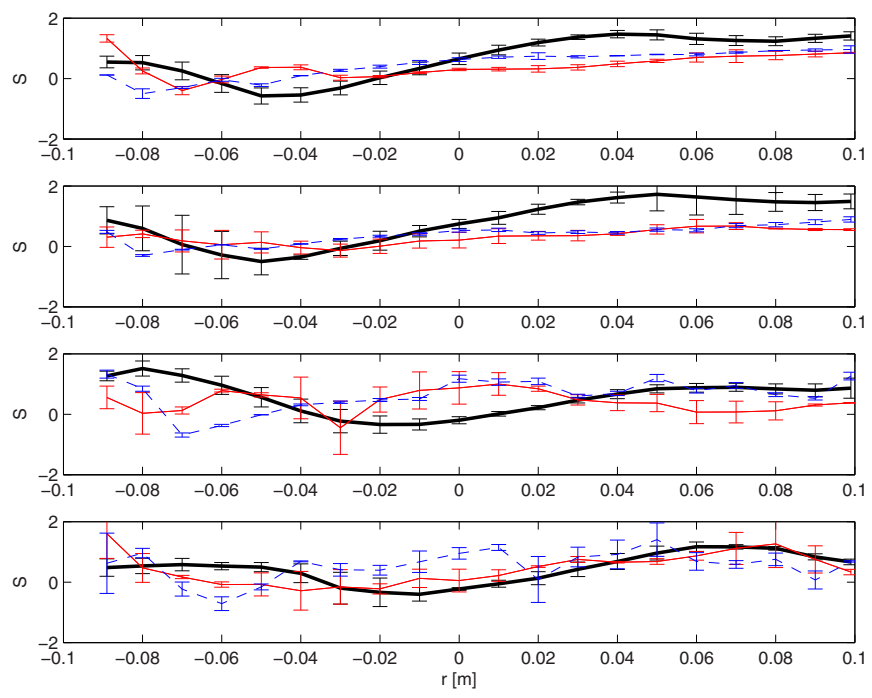

FIG. 6. (Color online) Same as in Fig. 1, for the $I_{\text {sat }}$ skewness radial profile at the vertical midplane.
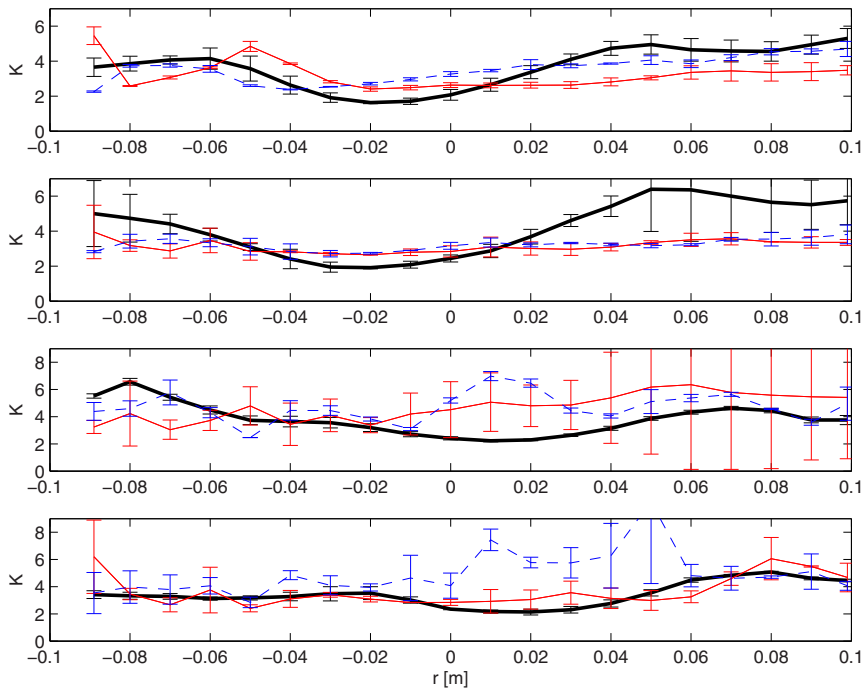

FIG. 7. (Color online) Same as in Fig. 1, for the $I_{\text {sat }}$ kurtosis radial profile at the vertical midplane.

$k_{\phi}=1$ to $k_{\phi}=0$ corresponds to the transition from ideal interchange to toroidally symmetric resistive interchange dynamics. ${ }^{6}$

In general, one observes that the agreement between three-dimensional simulations and experiments does not strongly depend on $N$. On the other hand, for the twodimensional simulations, the agreement strongly decreases with increasing $N$ for the majority of the observables. In fact, for $N=2$ and $N=4$ the agreement between two-dimensional simulations and experiments is comparable to the one observed in the case of three-dimensional simulations. The agreement decreases at higher $N$, and at $N=16$ all the observables reveal a complete disagreement. This points out that a three-dimensional model is essential to describe the transition from the ideal interchange regime to the resistive inter-
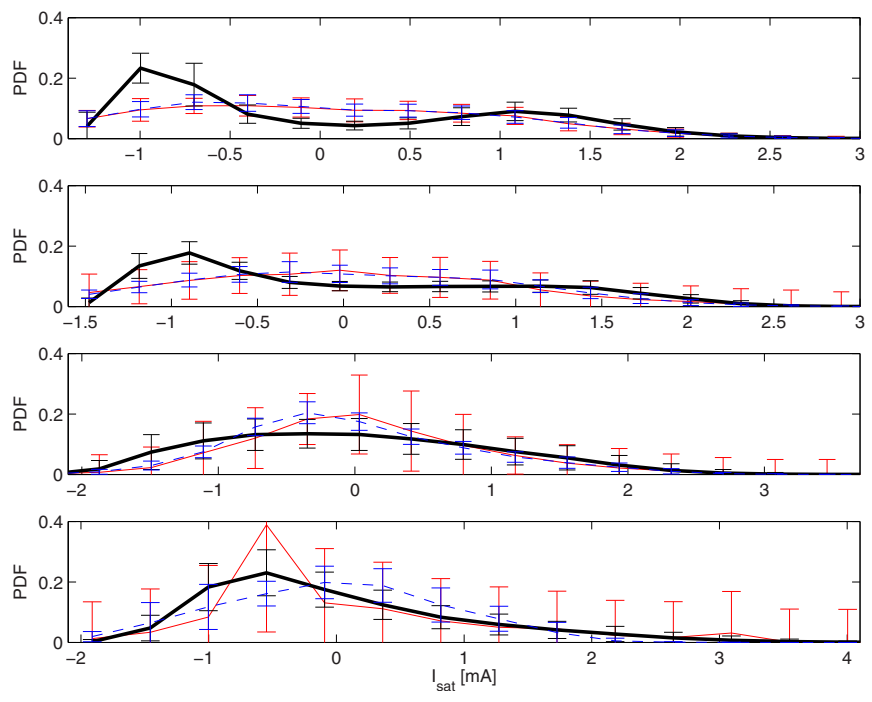

FIG. 8. (Color online) Same as in Fig. 1, for the probability distribution function of the $I_{\text {sat }}$ signal at the vertical midplane, at the radial point where $I_{\text {sat }}$ is equal to the $3 / 4$ of its peak value. 

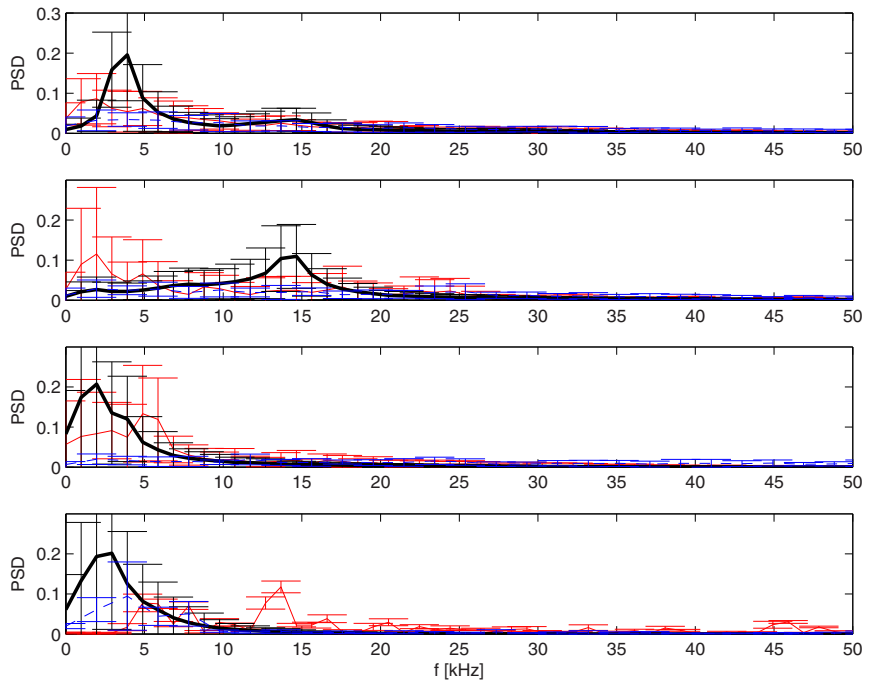

FIG. 9. (Color online) Same as in Fig. 1, for the power spectral density of the $I_{\text {sat }}$ signal at the vertical midplane and at the radial point where $I_{\text {sat }}$ is equal to the $3 / 4$ of its peak value.

change mode regime that has $k_{\|} \neq 0$ (not allowed in the twodimensional simulations), and therefore to model the plasma dynamics at high $N$.

\section{B. Metric}

The values of $S$ used to evaluate the weight of each observable in the global metric are listed in Table I. In general, uncertainty is relatively low (i.e., $S_{j}$ is close to 1 ), except in the case of the $k_{z}$ and $I_{\text {sat }}$ PDF and PSD observables. We also note that all the observables considered herein are at the second level of the comparison hierarchy; thus, $H=0.5$ in all cases.

The values of $\chi$ relevant to our validation project are plotted in Figs. 11 and 12, which describe the dependence of the simulation/experiment agreement as a function of $N$ for both the two-dimensional and three-dimensional simulations.
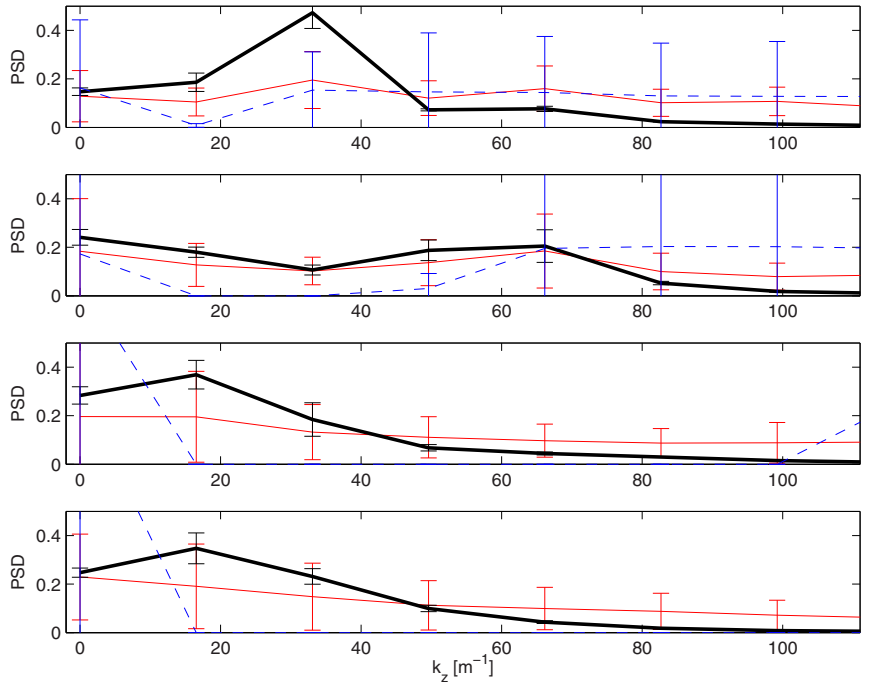

FIG. 10. (Color online) Same as in Fig. 1, for the power spectral density of the $I_{\text {sat }}$ signal as a function of the vertical wavenumber, at the vertical midplane and at the radial point where $I_{\text {sat }}$ is equal to the $3 / 4$ of its peak value.

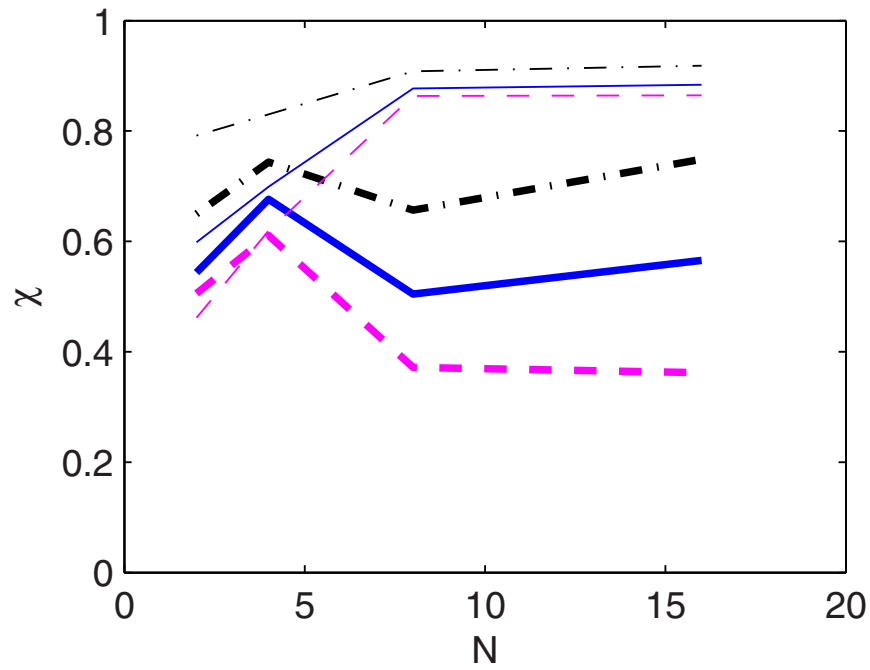

FIG. 11. (Color online) Values of the metric $\chi$ as a function of $N$ for different values of the parameter $d_{0}$ present in Eq. (2). Thick lines refer to the three-dimensional simulations, and thin lines refer to the twodimensional simulations. The cases $d_{0}=1$ (dash-dotted black lines), $d_{0}=1.5$ (solid blue lines), and $d_{0}=2$ (dashed magenta lines) are considered. In all cases $\lambda=0.5$.

In order to study the sensitivity of $\chi$ on the parameters present in Eq. (2), we plot in Fig. 11 the values of $\chi$ for different values of $d_{0}$, while in Fig. 12 we display the $\chi$ dependence on $\lambda$. It is shown that the trends are robust to the somewhat arbitrary choice of those parameters.

For the three-dimensional simulations, $\chi \simeq 0.5$, showing that the three-dimensional simulations equally well represent low and high $N$ scenarios. Reflecting on the observations presented when considering the agreement of each observable, a clear trend is instead observed in the case of the two-dimensional simulations, where the agreement decreases with $N$, passing from $\chi \simeq 0.6$ for $N=2$ to $\chi \simeq 0.9$ (i.e., almost complete disagreement) for $N=16$.

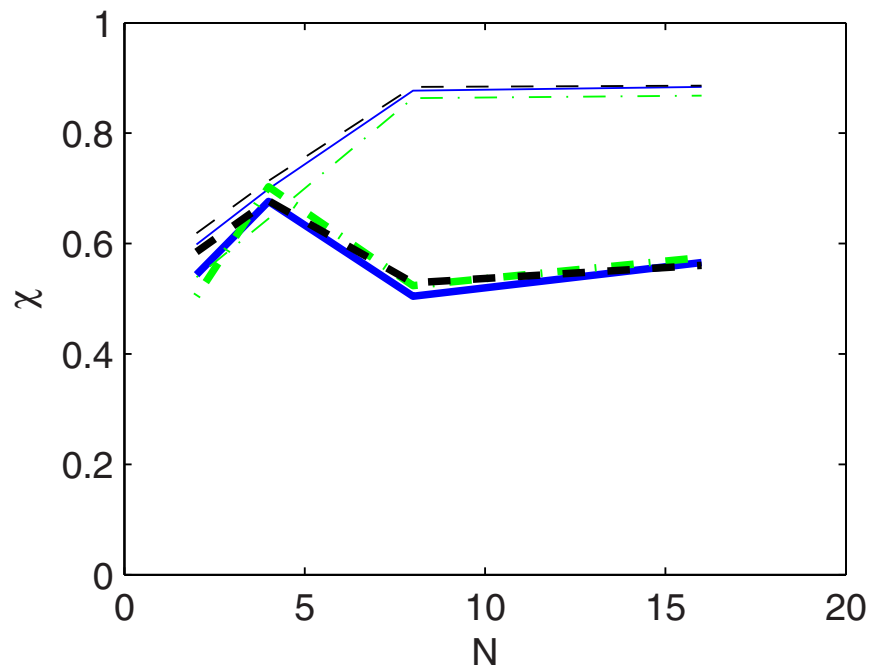

FIG. 12. (Color online) Values of the metric $\chi$ as a function of $N$ for different values of the parameter $\lambda$ present in Eq. (2). Thick lines refer to the three-dimensional simulations, and thin lines refer to the two-dimensional simulations. The cases $\lambda=0.1$ (dashed black lines), $\lambda=0.5$ (solid blue lines), and $\lambda=1$ (dash-dotted green lines) are considered. In all cases $d_{0}=1.5$. 


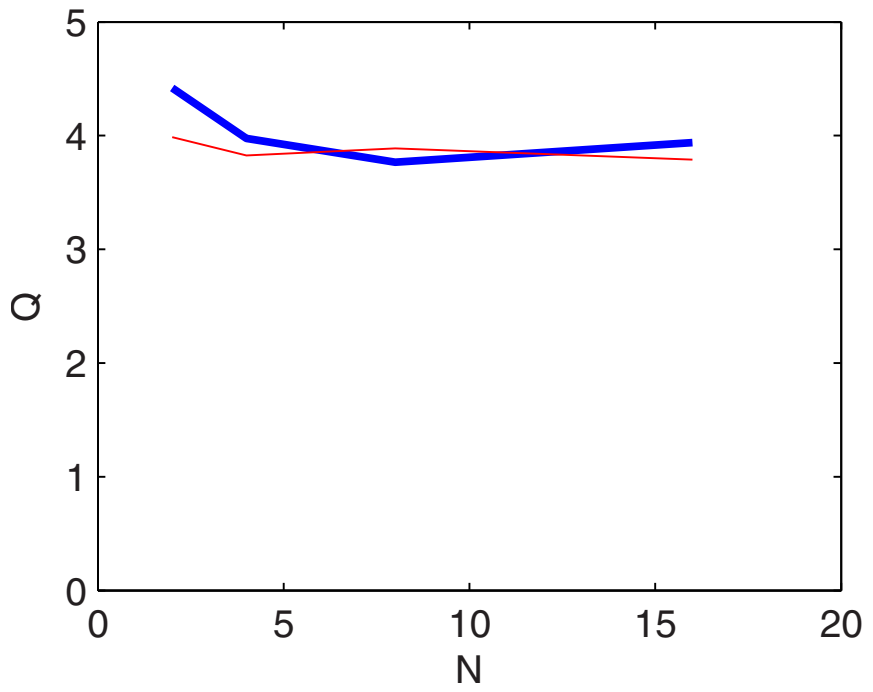

FIG. 13. (Color online) Validation quality $Q$ as a function of $N$ for twodimensional (thin red line) and three-dimensional (thick blue line) simulations.

\section{Quality}

The quality of the comparison is plotted in Fig. 13. Since the uncertainties for all the observables are relatively small and all the observables are at the second level of the validation hierarchy, $Q$ is about constant as a function of $N$, and in particular $Q \simeq 4$. The $Q$ values reported in the present validation project can be compared with $Q$ that would be obtained in a validation carried out by comparing exclusively the agreement of the experimental and simulation particle fluxes, that is, $Q \leq 0.25$.

In the analysis of the present example we do not split the quality of the comparison among observables with different dimensionalities. We only note that, with the exception of $k_{\phi}$, all the observables considered herein are one dimensional.

\section{CONCLUSIONS}

In the present paper we discussed the methodology for plasma turbulence code validation, focusing in particular on the assessment of the agreement between simulation and experiment. The assessment of such agreement starts at the level of each observable. We have pointed out that the distance between the experimental and simulation values should be evaluated and normalized to their uncertainties. Then, the agreement of each observable should be weighted into the global metric $\chi$, and care should be taken to reduce the weight of observables higher in the comparison hierarchy and with higher levels of uncertainties. The metric $\chi$ has been normalized in order to be equal to zero in the case of perfect agreement and 1 in the case of complete disagreement. A validation is not concluded until the quality of the comparison, $Q$, is also provided. The parameter $Q$ is an index that can be used to compare validations among them; it reveals how well a comparison has been made, indicating the number of observables used for the comparison and how constraining they are. A validation table should also be provided in order to allow a complete picture of the comparison.
The proposed methodology has been tested on the simulation of the basic plasma physics experiment TORPEX, focusing on measurements from Langmuir probes. We have considered the validation of a two-dimensional and a threedimensional code. The validation table is presented in Table I, while the values of $\chi$ and $Q$ are displayed in Figs. 11-13.

Can we conclude that the codes are validated successfully and able to describe TORPEX turbulence? The most immediate results of our validation exercise is that the threedimensional model is largely preferable than the twodimensional one in the high $N$ regime, where $k_{\|} \neq 0$, i.e., $\chi \simeq 0.5$ for the three-dimensional code, while $\chi \simeq 0.9$ for the two-dimensional one. In the low $N$ regime, where turbulence has $k_{\|}=0$, the two-dimensional and the three-dimensional models have a similar level of agreement, i.e., $\chi \simeq 0.5$. Establishing if the observed level of agreement is acceptable or not, i.e., if the code is validated or not, depends on the specific purpose of the code. The study of the validation table shows that, while equilibrium profiles and the nature of the main instabilities are reasonably well described by the threedimensional code, some turbulence details are not well modeled. Particularly important is the saturation level, which is underestimated by the simulations by a factor of 2 . For example, if the goal of our code is to predict the turbulence level, then most probably it will not be acceptable (unless one accepts a large error bar). Instead, if the code is to be used to predict the vertical mode number, $k_{z}$, it will most probably be acceptable (unless one needs an extremely high accuracy).

The example shows that it is relatively easy to use this methodology to discriminate among models and assess whether or not they follow the right trend. On the other hand, it is much more delicate to judge a single model in absolute terms.

\section{ACKNOWLEDGMENTS}

Financial support from the Fonds National Suisse de la Recherche Scientifique is acknowledged.

\section{APPENDIX A: THE EXPERIMENTAL SETUP}

The considered experiments are performed in TORPEX, a toroidal device with major radius $R=1 \mathrm{~m}$ and minor radius $a=0.2 \mathrm{~m}$. The vertical and toroidal components of the magnetic field create helicoidal field lines that terminate on the torus vessel. A hydrogen plasma is produced and sustained by microwaves in the electron cyclotron (EC) range of frequencies (a microwave power of $300 \mathrm{~W}$ is used in the experiments described here). Using the technique discussed in Ref. 12, it is observed that the plasma production is localized at the EC and UH layers, which are vertically elongated approximately around $r=-13 \mathrm{~cm}$ and $r=-2 \mathrm{~cm}$, respectively. Although in some recent experiments a limiter has been inserted in the TORPEX device (see, e.g., Ref. 13), no limiter is used in the experiment considered herein.

A toroidal magnetic field $B_{\phi}=76 \mathrm{mT}$ on axis is used, with four values of vertical magnetic field, $B_{z}=2.4,1.2,0.6,0.3 \mathrm{mT}$. This results in $\Delta=20,10,5$, and $2.5 \mathrm{~cm}$, corresponding to $N=2,4,8$, and 16 turns of a mag- 
netic field line in the device. Typical plasma parameters are in the ranges $n \simeq 10^{16} \mathrm{~m}^{-3}, T_{e} \simeq 5 \mathrm{eV}$, and $T_{i} \ll T_{e}$.

Diagnostics of the plasma dynamics used here include a set of stainless-steel pins, encased in a ceramic holder: ${ }^{14}$ (i) the SLP array, a linear array of eight Langmuir probes, with cylindrical tips having $1.5 \mathrm{~mm}$ diameter and $4 \mathrm{~mm}$ length and with $1.8 \mathrm{~cm}$ distance between tips, used to obtain most of the experimental results showed here; (ii) the HEXTIP array, a two-dimensional hexagonal Langmuir probe array covering the whole poloidal cross section, with spatial resolution of $3.5 \mathrm{~cm},{ }^{15}$ used to obtain the $k_{z}$ measurements; and (iii) TWIN, two identical Langmuir probes, separated toroidally by $180^{\circ}$, used to obtain the measurement of the toroidal mode number. We note that the $I_{\text {sat }}$ measurements are obtained by using the Langmuir probes in the $I_{\text {sat }}$ regime. For this purpose, a bias of $-40 \mathrm{~V}$ is used. On the other hand, the values of $\bar{n}, \bar{T}_{e}$, and $\bar{\phi}$ are obtained by sawtooth sweeping of the bias voltage of the Langmuir probes from -40 to $20 \mathrm{~V}$ with a $330 \mathrm{~Hz}$ frequency and fitting the obtained points with an I-V curve. A discussion on the interpretation of Langmuir probe data together with experimental consideration relative to the TORPEX device can be found in Ref. 16 .

\section{APPENDIX B: THE SIMULATION MODELS}

Owing to the low TORPEX plasma temperature, the drift-reduced Braginskii equations (see, e.g., Ref. 17) can be used to model the TORPEX plasma dynamics. In the limit of $T_{i} \ll T_{e}$ and $\beta \ll 1$, and assuming that $B_{z} \ll B_{\phi}$ so that $B \simeq B_{0} R /(R+r)$, since the magnetic curvature is constant along a field line and equal to $R+r$, these equations can be written as

$$
\begin{gathered}
\frac{\partial n}{\partial t}=\frac{c}{B}[\phi, n]+\frac{2 c}{e R B}\left(\frac{\partial p_{e}}{\partial z}-e n \frac{\partial \phi}{\partial z}\right)-\frac{\partial\left(n V_{\| e}\right)}{\partial x_{\|}}+S_{n}, \\
\frac{\partial \nabla_{\perp}^{2} \phi}{\partial t}=\frac{c}{B}\left[\phi, \nabla_{\perp}^{2} \phi\right]-V_{\| i} \frac{\partial \nabla_{\perp}^{2} \phi}{\partial x_{\|}}+\frac{2 B}{c m_{i} R n} \frac{\partial p_{e}}{\partial z} \\
+\frac{m_{i} \Omega_{c i}^{2}}{e^{2} n} \frac{\partial j_{\|}}{\partial x_{\|}}, \\
\frac{\partial T_{e}}{\partial t}=\frac{c}{B}\left[\phi, T_{e}\right]+\frac{4 c}{3 e R B}\left(\frac{7}{2} T_{e} \frac{\partial T_{e}}{\partial z}+\frac{T_{e}^{2}}{n} \frac{\partial n}{\partial z}-T_{e} \frac{\partial \phi}{\partial z}\right) \\
+\frac{2}{3} \frac{T_{e}}{e n} 0.71 \frac{\partial j_{\|}}{\partial x_{\|}}-\frac{2}{3} T_{e} \frac{\partial V_{\| e}}{\partial x_{\|}}-V_{\| e} \frac{\partial T_{e}}{\partial x_{\|}}+S_{T}, \\
\frac{\partial V_{\| i}}{\partial t}=\frac{c}{B}\left[\phi, V_{\| i}\right]-V_{\| i} \frac{\partial V_{\| i}}{\partial x_{\|}}-\frac{1}{n m_{i}} \frac{\partial p_{e}}{\partial x_{\|}}, \\
\quad-1.71 n \frac{\partial T_{e}}{\partial x_{\|}}+\frac{e n}{\sigma_{\|}} j_{\|}, \\
m_{e} n \frac{\partial V_{\| e}}{\partial t}=m_{e} n \frac{c}{B}\left[\phi, V_{\| e}\right]-m_{e} n V_{\| e} \frac{\partial V_{\| e}}{\partial x_{\|}}-T_{e} \frac{\partial n}{\partial x_{\|}}+e n \frac{\partial \phi}{\partial x_{\|}}
\end{gathered}
$$

where $\quad p_{e}=n T_{e}, \quad[a, b]=\partial_{r} a \partial_{z} b-\partial_{z} a \partial_{r} b, \quad j_{\|}=e n\left(V_{\| i}-V_{\| e}\right)$,
$\Omega_{c i}=e B /\left(m_{i} c\right)$, and $S_{n}$ and $S_{T}$ are the density and temperature sources. The $r$ coordinate denotes the radial direction, $x_{\|}$is parallel to $B$, and $z$ is the direction perpendicular to $r$ and $x_{\|}$(for $B_{v} \ll B_{\varphi}$ the vertical and $z$ directions are approximately the same).

We solve Eqs. (B1)-(B5) on a field-aligned grid using a finite difference scheme with Runge-Kutta time stepping and small diffusion terms. The computational domain has an annular shape with a cross section $r=-L_{r} / 2$ to $r=L_{r} / 2$ and $z=0$ to $z=L_{z}$. At $r=-L_{r} / 2$ and $r=L_{r} / 2$, Dirichlet boundary conditions are used for $n, T_{e}, \phi$, and $\nabla_{\perp}^{2} \phi$ and Neumann boundary conditions for $V_{\|_{e}}$ and $V_{\| i}$. For the parallel velocities we use Bohm boundary conditions $V_{\| i}= \pm c_{s}$ and $V_{\| e}= \pm c_{s} \exp \left(\underline{\left.\Lambda-e \phi / T_{e}\right)}\right.$ at the upper and lower walls, with $\Lambda=\log \sqrt{m_{i} /\left(2 \pi m_{e}\right)}$. To evaluate the effect of the boundary conditions on the simulation results, at $z=0$ and $z=L_{z}$ we explore both Dirichlet and Robin boundary conditions for $n$ and $T_{e}$, and for $\phi$ we use both Dirichlet boundary conditions $e \phi=\Lambda T_{e}$ (implying $V_{\| e}=V_{\| i}$ ) and a boundary condition of the form $\partial_{z} \phi \propto\left(e \phi-\Lambda T_{e}\right)$. We have used source profiles that mimic the $\mathrm{EC}$ and $\mathrm{UH}$ resonance layers in TORPEX, i.e., $S_{n, T}=S_{0 ; n, T}\left\{S_{\mathrm{UH}} \exp \left[-\left(r-r_{\mathrm{UH}}\right)^{2} / \lambda_{\mathrm{UH}}^{2}\right]\right.$ $\left.+S_{\mathrm{EC}} \exp \left[-\left(r-r_{\mathrm{EC}}\right)^{2} / \lambda_{\mathrm{EC}}^{2}\right]\right\}$, with $S_{\mathrm{UH}}=1.5, \quad S_{\mathrm{EC}}=1, \quad \lambda_{\mathrm{UH}}$ $=1 \mathrm{~cm}, \lambda_{\mathrm{EC}}=0.5 \mathrm{~cm}, r_{\mathrm{UH}}=-2 \mathrm{~cm}, r_{\mathrm{EC}}=-6 \mathrm{~cm}$, and values of the source strength $\left(S_{0 n}=1.5 \times 10^{20} \mathrm{~m}^{-3} \mathrm{~s}^{-1}, S_{0 T}=3.5\right.$ $\times 10^{4} \mathrm{eV} / \mathrm{s}$ ) estimated experimentally through a global balance of the TORPEX plasma. We remark that dependence of the UH resonance position on $n$ is neglected in the present model. Other values used are $R=1 \mathrm{~m}, m_{i} / m_{e}=200$, $\Lambda=3$, and the resistivity has been varied in the range $\nu=(0.01-1) c_{s} / R$ in order to check the influence of this parameter.

If only $k_{\|} \simeq 0$ modes are considered, simple twodimensional fluid equations that describe the plasma turbulence can be considered. The Braginskii equations are integrated in the parallel direction in order to evolve the line-integrated density, $n(r, z)=\int n\left(r, z, x_{\|}\right) d x_{\|} / L_{c}$; potential, $\phi(r, z)=\int \phi\left(r, z, x_{\|}\right) d x_{\|} / L_{c} ;$ and temperature, $T_{e}(r, z)$ $=\int T_{e}\left(r, z, x_{\|}\right) d x_{\|} / L_{c}$, with $L_{c}=2 \pi N R$ being the magnetic field line length. We use Bohm's boundary conditions to take into account the ion and electron parallel flows at the sheath edge. By assuming that the density at the edge is equal to $n(r, z) / 2$, it is possible to approximate the ion and electron flows as $\Gamma_{\|, i}=n c_{s} / 2$ and $\Gamma_{\|, e}=n c_{s} \exp \left(-e \phi / T_{e}+\Lambda\right) / 2$. The evolution equations for $n, \phi$, and $T_{e}$ thus become

$$
\begin{gathered}
\frac{\partial n}{\partial t}=\left[\frac{c \phi}{B_{0}}, n\right]+\frac{2 c}{e R B_{0}}\left(n \frac{\partial T_{e}}{\partial z}+T_{e} \frac{\partial n}{\partial z}-e n \frac{\partial \phi}{\partial z}\right) \\
-\frac{\sigma n c_{s}}{R} \exp \left(\Lambda-e \phi / T_{e}\right)+S_{n} \\
\frac{\partial \nabla^{2} \phi}{\partial t}=\left[\frac{c \phi}{B_{0}}, \nabla^{2} \phi\right]+\frac{2 B_{0}}{c m_{i} R}\left(\frac{T_{e}}{n} \frac{\partial n}{\partial z}+\frac{\partial T_{e}}{\partial z}\right) \\
+\frac{\sigma c_{s} m_{i} \Omega_{i}^{2}}{e R}\left[1-\exp \left(\Lambda-e \phi / T_{e}\right)\right],
\end{gathered}
$$




$$
\begin{aligned}
\frac{\partial T_{e}}{\partial t}= & {\left[\frac{c \phi}{B_{0}}, T_{e}\right]+\frac{4 c}{3 e R B_{0}}\left(\frac{7}{2} T_{e} \frac{\partial T_{e}}{\partial z}+\frac{T_{e}^{2}}{n} \frac{\partial n}{\partial z}-e T_{e} \frac{\partial \phi}{\partial z}\right) } \\
& -\frac{2}{3} \frac{\sigma T_{e} c_{s}}{R}\left[1.71 \exp \left(\Lambda-e \phi / T_{e}\right)-0.71\right]+S_{T},
\end{aligned}
$$

where $\sigma=R / L_{c}=\Delta /\left(2 \pi L_{v}\right)$. We note that a similar system of equations has been used in Ref. 18. The system of equations (B6)-(B8) has been solved numerically, using a numerical code developed from the ESEL code. ${ }^{19}$ The algorithm used is described in Ref. 20.

For the two-dimensional simulations, we consider a domain with extension $L_{r}$ in the radial direction and $\Delta$ along $z$. The boundary conditions are periodic along the vertical direction (due to the flute property of the interchange mode), and we use Dirichlet boundary conditions in the radial direction. In order to study the sensitivity of the results to the parallel boundary conditions, a scan of the $\sigma$ parameter has been performed.

Both for the three-dimensional and two-dimensional cases the simulation is started from random noise. Then, the sources introduce plasma and heat, increasing the plasma pressure and triggering the interchange instability. The interchange instability leads to density and particle transport in the radial direction from the source region to the low field side of the machine. At the same time, plasma is removed from the system by parallel losses. The results discussed in the present paper focus on the quasi-steady-state period, established after the initial simulation transient, as a result of a balance between parallel losses, perpendicular transport, and sources. A detailed analysis of the plasma dynamics described by the three-dimensional model (B1)-(B5) has been presented in Refs. 5 and 6, while the simulation results obtained from the two-dimensional model (B6)-(B8) have been discussed in Ref. 7.

${ }^{1}$ P. W. Terry, M. Greenwald, J.-N. Leboeuf, G. R. McKee, D. R. Mikkelsen, W. M. Nevins, D. E. Newman, D. P. Stotler, Task Group on Verification and Validation, U.S. Burning Plasma Organization, and U.S. Transport Task Force, Phys. Plasmas 15, 062503 (2008); M. Greenwald, ibid. 17, 058101 (2010).

${ }^{2}$ A. M. Dimits, G. Bateman, M. A. Beer, B. I. Cohen, W. Dorland, G. W. Hammett, C. Kim, J. E. Kinsey, M. Kotschenreuther, A. H. Kritz, L. L.
Lao, J. Mandrekas, W. M. Nevins, S. E. Parker, A. J. Redd, D. E. Shumaker, R. Sydora, and J. Weiland, Phys. Plasmas 7, 969 (2000); J. Birn, J. F. Drake, M. A. Shay, B. N. Rogers, R. E. Denton, M. Hesse, M. Kuznetsova, Z. W. Ma, A. Bhattacharjee, A. Otto, and P. L. Pritchett, J. Geophys. Res. 106, 3715, doi:10.1029/1999JA900449 (2001); P. Ricci, J. U. Brackbill, W. Daughton, and G. Lapenta, Phys. Plasmas 11, 4102 (2004); V. Umansky, M. S. Day, and T. D. Rognlien, Numer. Heat Transfer, Part B 47, 533 (2005); J. R. Myra, D. A. D’Ippolito, D. A. Russel, and M. Umansky, Bull. Am. Phys. Soc. 52, 533 (2007).

${ }^{3}$ P. Ricci, C. Theiler, A. Fasoli, I. Furno, B. Labit, S. H. Müller, M. Podestà, and F. M. Poli, Phys. Plasmas 16, 055703 (2009).

${ }^{4}$ A. Fasoli, B. Labit, M. McGrath, S. H. Müller, G. Plyushchev, M. Podestà, and F. M. Poli, Phys. Plasmas 13, 055902 (2006); A. Fasoli, A. Burckel, L. Federspiel, I. Furno, K. Gustafson, D. Iraji, B. Labit, J. Loizu, G. Plyushchev, P. Ricci, C. Theiler, A. Diallo, S. H. Müller, M. Podestà, and F. M. Poli, Plasma Phys. Controlled Fusion 52, 124020 (2010).

${ }^{5}$ P. Ricci and B. N. Rogers, Phys. Plasmas 16, 092307 (2009).

${ }^{6}$ P. Ricci and B. N. Rogers, Phys. Rev. Lett. 104, 145001 (2010).

${ }^{7}$ P. Ricci, B. N. Rogers, and S. Brunner, Phys. Rev. Lett. 100, 225002 (2008); P. Ricci and B. N. Rogers, Phys. Plasmas 16, 062303 (2009).

${ }^{8}$ R. H. Myers, D. C. Montgomery, and C. M. Anderson-Cook, Response Surface Methodology: Process and Product Methodology, Using Designed Experiment, 3rd ed. (Wiley, New York, 2009).

${ }^{9}$ F. M. Poli, P. Ricci, A. Fasoli, and M. Podestà, Phys. Plasmas 15, 032104 (2008).

${ }^{10}$ B. Labit, I. Furno, A. Fasoli, A. Diallo, S. H. Müller, G. Plyushchev, M. Podestà, and F. M. Poli, Phys. Rev. Lett. 98, 255002 (2007); B. Labit, A. Diallo, A. Fasoli, I. Furno, D. Iraji, S. H. Müller, G. Plyushchev, M. Podestà, F. M. Poli, P. Ricci, C. Theiler, and J. Horacek, Plasma Phys. Controlled Fusion 49, B281 (2007).

${ }^{11}$ F. M. Poli, S. Brunner, A. Diallo, A. Fasoli, I. Furno, B. Labit, S. H. Müller, G. Plyushchev, and M. Podestà, Phys. Plasmas 13, 102104 (2006).

${ }^{12}$ M. Podestà, A. Fasoli, B. Labit, M. McGrath, S. H. Müller, and F. M. Poli, Plasma Phys. Controlled Fusion 48, 1053 (2006).

${ }^{13}$ C. Theiler, I. Furno, P. Ricci, A. Fasoli, B. Labit, S. H. Müller, and G. Plyushchev, Phys. Rev. Lett. 103, 065001 (2009).

${ }^{14}$ M. Podestà, Ph.D. thesis, Ecole Polythenique Federale de Lausanne, 2007; http://library.epfl.ch/en/theses/?nr $=3765$

${ }^{15}$ S. H. Müller, A. Fasoli, B. Labit, M. McGrath, O. Pisaturo, G. Plyushchev, M. Podestà, and F. M. Poli, Phys. Plasmas 12, 090906 (2005).

${ }^{16}$ C. Theiler, I. Furno, A. Kuenlin, Ph. Marmillod, and A. Fasoli, Rev. Sci. Instrum. 82, 013504 (2011).

${ }^{17}$ A. Zeiler, J. F. Drake, and B. Rogers, Phys. Plasmas 4, 2134 (1997).

${ }^{18}$ N. Bisai, A. Das, S. Deshpande, R. Jha, P. Kaw, A. Sen, and R. Singh, Phys. Plasmas 11, 4018 (2004).

${ }^{19}$ V. Naulin, J. Nycander, and J. J. Rasmussen, Phys. Rev. Lett. 81, 4148 (1998); O. E. Garcia, V. Naulin, A. H. Nielsen, and J. J. Rasmussen, ibid. 92, 165003 (2004).

${ }^{20}$ V. Naulin and A. H. Nielsen, SIAM J. Sci. Comput. (USA) 25, 104 (2003). 